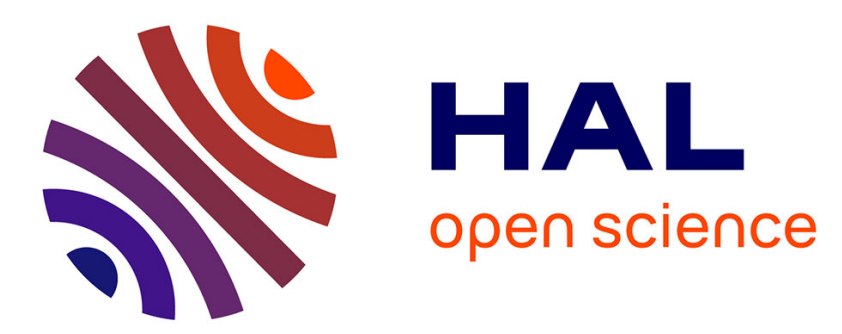

\title{
Delay-Scheduled State-Feedback Design for Time-Delay Systems with Time-Varying Delays - A LPV Approach
}

Corentin Briat, Jean-François Lafay, Olivier Sename

\section{To cite this version:}

Corentin Briat, Jean-François Lafay, Olivier Sename. Delay-Scheduled State-Feedback Design for Time-Delay Systems with Time-Varying Delays - A LPV Approach. Systems and Control Letters, 2009, 58, pp.664-671. 10.1016/j.sysconle.2009.06.001 . hal-00402238

\section{HAL Id: hal-00402238 \\ https://hal.science/hal-00402238}

Submitted on 28 Oct 2010

HAL is a multi-disciplinary open access archive for the deposit and dissemination of scientific research documents, whether they are published or not. The documents may come from teaching and research institutions in France or abroad, or from public or private research centers.
L'archive ouverte pluridisciplinaire HAL, est destinée au dépôt et à la diffusion de documents scientifiques de niveau recherche, publiés ou non, émanant des établissements d'enseignement et de recherche français ou étrangers, des laboratoires publics ou privés. 


\title{
Delay-Scheduled State-Feedback Design for Time-Delay Systems with Time-Varying Delays - A LPV Approach
}

\author{
C. Briat ${ }^{a}$ O. Sename ${ }^{a}$ J.F. Lafay ${ }^{b}$ \\ ${ }^{\mathrm{a}}$ GIPSA-Lab, Department of Control Systems (former LAG), Grenoble Universités, ENSE ${ }^{3}$ - BP46, 38402 Saint Martin d'Hères - Cedex \\ FRANCE, \{Corentin.Briat, Olivier.Sename\}@gipsa-lab.inpg.fr \\ ${ }^{\mathrm{b}}$ IRCCyN - Centrale de Nantes, 1 rue de la Noë - BP 92101, 44321 Nantes Cedex 3 - FRANCE, Jean-Francois.Lafay@irccyn.ec-nantes.fr
}

\begin{abstract}
This paper is concerned with the synthesis of delay-scheduled state-feedback controllers which stabilize linear systems with timevarying delays. In this framework, it is assumed that the delay is approximately known in real-time and used in the controller in a scheduling fashion. First, a new model transformation turning a time-delay system into an uncertain LPV system is introduced. Using this transformation, a new delay-dependent stability test based on the so-called full block $\mathcal{S}$-procedure is developed and from this result, a new delay-dependent stabilization result is derived. Since the resulting LMI conditions depend polynomially on the parameters, a relaxation result is then applied in order to obtain a tractable finite set of finite-dimensional LMIs. The interests of the approach resides in 1) the synthesis of a new type of controllers scheduled by the delay value which has a lower memory consumption than controllers with memory (since it is not necessary to store past values of the state), and 2) an easy consideration of uncertainties on the delay knowledge.
\end{abstract}

Key words: Time-delay systems, Linear parameter varying systems, Robust linear matrix inequalities, Relaxation, Convex optimization

\section{Introduction}

Since several years, time-delay systems (TDS) have been intensively studied (Niculescu, 2001; Fridman and Shaked, 2002; Gu et al., 2003; Jiang and Han, 2005; Fridman, 2006; Gouaisbaut and Peaucelle, 2006a; Suplin et al., 2006; Xu et al., 2006; Kao and Rantzer, 2007). It has been shown that delays are often responsible of instability and poor performances and thus, they should not be neglected when analyzing system stability or synthesizing control laws. Since the advent of communication networks and embedded electronics, systems with time-varying delays have attracted more and more interest. Indeed, a communication network can be viewed as a communication channel inducing delays depending on the load of the network which varies in time.

In some applications, it may be possible to measure or compute the delay from a mathematical model and in this case, it could be interesting to use this information in the controller. In (Witrant et al., 2005), a predictive approach to control Network Controlled Systems is given but a network model is necessary to compute the prediction horizon. In (Sename et al., 1995) a state feedback with internal delay is designed but the robustness issue w.r.t delay measure- ment uncertainties is not considered. The authors proposed in (Briat et al., 2007) some preliminary results on a new control design technique for TDS. Using a new model transformation, the time-delay system is transformed into an uncertain Linear Parameter Varying (LPV) system where the delay acts as a parameter. However the results stand for interval delay (with non zero delay) only. Some interesting results on TDS based on Linear Fractional Transformation (LFT) may also be found in (Zhang et al., 2001; Roozbehani and Knospe, 2005; Gouaisbaut and Peaucelle, 2006b; Kao and Rantzer, 2007).

Following the preliminary results in (Briat et al., 2007), the LPV/uncertain system stability analysis and control synthesis tools are used to prove stability and stabilize timedelay systems (See (Apkarian and Adams, 1998; Scherer, 1999)). The main contributions of the paper are:

- Following the idea of (Briat et al., 2007), a new model transformation which corrects some weaknesses of the previous one is introduced.

- Using these results, a delay-dependent stability with guaranteed $\mathcal{L}_{2}$ performances test is provided. It is obtained using the so-called full-block $\mathcal{S}$-procedure and is expressed through parameter dependent LMI conditions. Computational approximations are then used in order 
to make the problem tractable by numerical procedures. - From this stability test a stabilization result is derived. The obtained controller is smoothly scheduled by an approximate delay value and the error on the delay knowledge is taken into account as a robustness constraint.

In this paper, the following systems are considered

$$
\begin{aligned}
& \dot{x}(t)=A x(t)+A_{h} x(t-h(t))+B_{u} u(t)+E w(t) \\
& z(t)=C x(t)+C_{h} x(t-h(t))+D_{u} u(t)+F w(t)
\end{aligned}
$$

where $x \in \mathbb{R}^{n}, u \in \mathbb{R}^{m}, w \in \mathbb{R}^{p}$ and $z \in \mathbb{R}^{q}$ are respectively the system state, the control input, the exogenous input and the controlled output.

The time-varying delay $h(t)$ is assumed to belong to the set

$$
\mathscr{H}:=\left\{h \in \mathcal{C}^{1}\left(\mathbb{R}_{+}, H\right), \dot{h}: \mathbb{R}_{+} \rightarrow U\right\}
$$

where $\mathcal{C}^{1}(I, J)$ denotes the set of continuous functions with continuous derivative mapping $I$ to $J, H:=\left[h_{\min }, h_{\max }\right]$ and $U:=\left[\mu_{\min }, \mu_{\max }\right]$. The delay takes then bounded values and has a bounded derivative.

It is convenient to introduce the following set of vertices

$$
\begin{aligned}
& \mathcal{V}_{h}:=\left\{h_{\min }, h_{\max }\right\} \\
& \mathcal{V}_{\mu}:=\left\{\mu_{\min }, \mu_{\max }\right\} \quad \mu_{\max }<1
\end{aligned}
$$

The aim of the paper is to find delay-scheduled statefeedback controllers of the form

$$
u(t)=K(\hat{h}(t)) x(t)
$$

which stabilize system (1) and where $K(\cdot)$ may be a linear, polynomial or rational function of the known value of the delay $\hat{h}(t)=h(t)+\delta_{h}(t)$, with knowledge error $\delta_{h}(t)$ belonging to

$$
\Delta:=\left\{\delta_{h}: \mathbb{R}_{+} \rightarrow \Delta, \dot{\delta}_{h}: \mathbb{R}_{+} \rightarrow \Delta_{\nu}\right\}
$$

where $\Delta:=[-\delta, \delta]$ and $\Delta_{\nu}:=\left[\nu_{\min }, \nu_{\max }\right]$.

Finally we define the set of known delay values:

$$
\hat{\mathscr{H}}:=\left\{\hat{h} \in \mathcal{C}^{1}\left(\mathbb{R}_{+}, \hat{H}\right), \dot{\hat{h}}: \mathbb{R}_{+} \rightarrow \hat{U}\right\}
$$

with $\hat{H}:=H+\Delta$ and $\hat{U}:=U+\Delta_{\nu}$.

The notations are as follows, for symmetric matrices $A, B, A>B$ means $A-B$ is positive definite (i.e. $A-B>$ $0)$. For a square matrix $A$ we have $A^{\mathcal{S}}=A+A^{T}$ where $A^{T}$ is the transpose of $A . \operatorname{Ker}(A)$ is a basis of the nullspace of $A . A_{\perp}$ is any basis of the orthogonal complement of $\Im(A)$ (i.e. $A^{T} A_{\perp}=0$ ) and $\operatorname{Im}(A)$ is the image set of $A$. $\oplus$ is the direct sum of matrices: $A \oplus B=\left[\begin{array}{cc}A & 0 \\ 0 & B\end{array}\right] \cdot \mathcal{L}_{2}$ is the space of signals with finite energy (finite $\mathcal{L}_{2}$-norm): $\|f\|_{\mathcal{L}_{2}}^{2}:=\int_{0}^{+\infty}|f(t)|^{2} d t<+\infty$.

The paper is organized as follows, in Section 2 we introduce some preliminary results. In Section 3, the new model transformation and the associated comparison system are presented. The first main result of the paper: a new delay-dependent stability test based on the use of the model transformation is developed in Section 4. Finally, the second main result of the paper: the delay-scheduled state-feedback design is detailed in Section 5.

\section{Converting Polynomial into Linear Dependence}

This section is devoted to the presentation of a relaxation technique for polynomially parameter dependent LMI. The key idea is to use the Finsler's lemma (Skelton et al., 1997) to linearize the dependence on the parameters. The following definition will be useful in the sequel

Definition 2.1 A square matrix $S$ is said to be $\mathcal{S}^{2}$ structured if it writes $S=\left[S_{i j}\right]_{i, j}$ with blocks $S_{i j} \in$ $\mathbb{R}^{k \times k}, k>1$ such that

$$
S_{i j}:= \begin{cases}0_{k \times k} & \text { if } i=j \\ S_{i j}=S_{j i}^{T} \in \mathbb{K}^{k} & \text { if } i \neq j\end{cases}
$$

It is now possible to express the linearization lemma which has also been provided in (Sato, 2006; Sato and Peaucelle, 2007).

Lemma 2.1 Suppose that a $\mathcal{M}(\delta)$ is a polynomially parametrized symmetric matrix in $\delta \in \Delta$ admitting a spectral factorization $\mathcal{M}(\delta)=U^{T}(\delta) \mathcal{N} U(\delta)<0$ where $U(\delta)$ is a basis of polynomials. Then $\mathcal{M}(\delta)<0$ for every $\delta \in \Delta$ if there exists a matrix $\mathcal{P}$ and a $\mathcal{S}^{2}$-structured matrix $\mathcal{R}$ such that

$$
\mathcal{N}+\mathcal{R}+\mathcal{P} V(\delta)+V^{T}(\delta) \mathcal{P}^{T}<0 \quad \delta \in \Delta
$$

where $U(\delta)=\operatorname{Ker}(V(\delta))$ and $\mathcal{R}$ is $\mathcal{S}^{2}$-structured (i.e. $U^{T}(\delta) \mathcal{R} U(\delta)=0$ with $\mathcal{R}=\mathcal{R}^{T} \neq 0$ ).

Proof: The proof is given in Appendix A and relies on the Finsler's Lemma (Skelton et al., 1997).

Remark 2.1 If the matrix $V(\delta)$ is affine in $\delta$ then lemma 2.1 can be used as a linearization procedure to linearize polynomially parameter dependent LMIs. It can be shown that every polynomially parametrized LMI can be expressed through a basis $U(\delta)$ which leads to an affine matrix $V(\delta)$. Indeed, the trivial case $U(\delta)=\operatorname{col}\left(I, \delta_{1}, \ldots, \delta_{N}, \delta_{1}^{2}, \ldots\right)$ leads to an affine $V(\delta)$. Hence, assuming that a 'good' basis is used, then (7) is affine and thus can be treated as in the polytopic/affine framework (using multi-convexity).

This approach is well dedicated to small and medium size problems since the size and the number of LMIs grows very quickly depending on the degree of polynomials, the number of parameters and the size of the initial LMI. In the case of large size problems, it may be interesting to use the relaxation proposed in (Ben-Tal and Nemirovski, 2002; Scherer, 2006). It is worth mentioning that this result is highly related to the so-called Sum-of-Squares (SoS) relaxation of parameter dependent LMIs.

An important remark concerns the relaxation of LMIs with rational parameter dependence. This type of LMIs 
can indeed be turned into LMIs with polynomial dependence using the full-block $\mathcal{S}$-procedure (Scherer and Weiland, 2005) which can be, in turn, transformed into LMIs with linear dependence using lemma 2.1.

\section{A new model transformation}

A new model transformation allowing to turn a TDS with time-varying delays into an uncertain LPV system is provided in this section. The main advantages of this new operator compared to (Briat et al., 2007) is the consideration of zero delay values and a tighter computation of the $\mathcal{L}_{2}$ induced norm of the operator.

Define then the operator

$$
\begin{aligned}
\mathcal{D}_{h}: \mathcal{L}_{2} & \rightarrow \mathcal{L}_{2} \\
\eta(t) & \rightarrow \frac{1}{\sqrt{h(t) h_{\max }}} \int_{t-h(t)}^{t} \eta(s) d s
\end{aligned}
$$

Proposition 3.1 This operator enjoys the following properties:

(i) $\mathcal{D}_{h}$ is $\mathcal{L}_{2}$ input/output stable.

(ii) $\mathcal{D}_{h}$ has a $\mathcal{L}_{2}$ induced norm lower than 1.

Proof: The proof is given in Appendix B and is based on a similar method as of ( $\mathrm{Gu}$ et al., 2003).

The remaining of the section explains the use of the operator $\mathcal{D}_{h}$ in order to turn a time-delay system into an uncertain LPV system. Let us consider system (1) and note that $x_{h}(t)=x(t)-h(t) \mathcal{D}_{h}(\dot{x}(t))$. Thus, substituting this equality into system (1) yields

$$
\begin{aligned}
\dot{x}(t) & =\bar{A} x(t)-\alpha(t) A_{h} w_{0}(t)+B_{u} u(t)+E w(t) \\
z_{0}(t) & =\dot{x}(t) \\
z(t) & =\bar{C} x(t)-\alpha(t) C_{h} w_{0}(t)+D_{u} u(t)+F w(t) \\
w_{0}(t) & =\mathcal{D}_{h}\left(z_{0}(t)\right) \\
\alpha(t) & =\sqrt{h(t) h_{\max }} \\
\bar{A} & =A+A_{h} \quad \bar{C}=C+C_{h}
\end{aligned}
$$

which is an uncertain LPV system with state $x(t)$ expressed in 'LFT' form. Indeed, the system is

- uncertain due to the presence of the 'unknown' structured norm bounded LTV dynamic operator $\mathcal{D}_{h}$ and

- parameter varying due to the presence $\sqrt{h(t)}$ whose dependence is even affine.

In order to deal with the uncertain operator $\mathcal{D}_{h}$, robust stability and robust control theories based on the full-block $\mathcal{S}$-procedure approach will be considered (Scherer and Weiland, 2005). The parameter dependence will be tackled using parameter dependent Lyapunov functions.

Remark 3.1 According to (Gu et al., 2003), system (10) is not equivalent to (1) due to the use of the model transformation induced by the use of the operator $\mathcal{D}_{h}$. Without entering too much in details, the use of model transformation often introduces conservatism in the approach by adding additional dynamics to the comparison (the transformed) model which may be unstable even if the original system is stable. Hence the stability test performed on the comparison model (10) may fail even if the initial system (1) is stable. However, in the stabilization problem, this is less critical since it is aimed to stabilize the system and thus the additional dynamics will be implicitly stabilized.

\section{Delay-dependent stability}

In this section, we develop the first main result of the paper: a new delay dependent stability test based on the comparison model (10) obtained from system (1) used along with the model transformation presented in section 3 .

Lemma 4.1 System (10) without control input (i.e. $u(t)=$ $0)$ is asymptotically stable for $h \in \mathscr{H}$ and satisfies the $\mathcal{L}_{2}$ performance constraint $\|z\|_{\mathcal{L}_{2}} /\|w\|_{\mathcal{L}_{2}}<\gamma$ if there exist a smooth matrix function $P: H \rightarrow \mathbb{S}_{++}^{n}$, matrix functions $D: H \times U \rightarrow \mathbb{S}_{++}^{n}$ and a scalar $\gamma>0$ such that the $L M I$ (8) holds for all $\alpha \in\left[\sqrt{h_{\min } h_{\max }}, h_{\max }\right]$ and $\dot{h} \in \mathcal{V}_{\mu}$.

Proof: The proof is given in Appendix C. It is based on an application of the full-block $\mathcal{S}$-procedure to deal with the uncertain operator $\mathcal{D}_{h}$ while the parameters are considered through the use a parameter dependent Lyapunov.

A direct corollary can be obtained immediately by choosing all the parameter dependent matrices to be constant. Corollary 4.1 System (10) without control input (i.e. $u(t)=0)$ is quadratically asymptotically stable for $h \in \mathscr{H}$ and satisfies the $\mathcal{L}_{2}$ performance constraint $\left.\|z\|\right|_{\mathcal{L}_{2}} /\|w\|_{\mathcal{L}_{2}}<$ $\gamma$ if there exist matrices $P, D \in \mathbb{S}_{++}^{n}$ and a scalar $\gamma>0$ such that the LMI

$$
\left[\begin{array}{ccccc}
{\left[\bar{A}^{T} P\right]^{\mathcal{S}}} & -\alpha P A_{h} & P E & \bar{C}^{T} & \bar{A}^{T} D \\
\star & -D & 0 & -\alpha C_{h}^{T} & -\alpha A_{h}^{T} D \\
\star & \star & -\gamma I_{p} & F^{T} & E^{T} D \\
\star & \star & \star & -\gamma I_{q} & 0 \\
\star & \star & \star & \star & -D
\end{array}\right]<0
$$

holds for all $\alpha \in\left\{\sqrt{h_{\min } h_{\max }}, h_{\max }\right\}$.

Proof: The proof is straightforward application of lemma 4.1. Since the LMI is linear in $\sqrt{h}$ then using a convex argument it suffices to check the feasibility of the LMI at the vertices of $H$ (i.e. $\mathcal{V}_{h}$ ) to conclude on the stability over the whole set $H$.

The next corollary is obtained by fixing all parameter dependent matrices such that the resulting parameter dependent LMI is of maximum degree 2. It is then relaxed using Lemma 2.1.

Corollary 4.2 System (10) without control input (i.e. $u(t)=0)$ is asymptotically stable for $h \in \mathscr{H}$ and satisfies the $\mathcal{L}_{2}$ performance constraint $\|z\|_{\mathcal{L}_{2}} /\|w\|_{\mathcal{L}_{2}}<\gamma$ if there exist matrices $P_{0}, P_{1}, D_{0}, D_{1} \in \mathbb{S}^{n}$, a full matrix $\Theta$, a $\mathcal{S}^{2}$ - 


$$
\left[\begin{array}{ccccc}
{\left[\bar{A}^{T} P(h)\right]^{\mathcal{S}}+\frac{d P}{d h} \dot{h}-\alpha P(h) A_{h}} & P(h) E & \bar{C}^{T} & \bar{A}^{T} D(h, \dot{h}) \\
\star & -D(h, \dot{h}) & 0 & -\alpha C_{h}^{T} & -\alpha A_{h}^{T} D(h, \dot{h}) \\
\star & \star & -\gamma I_{p} & F^{T} & E^{T} D(h, \dot{h}) \\
\star & \star & \star & -\gamma I_{q} & 0 \\
\star & \star & \star & \star & -D(h, \dot{h})
\end{array}\right]<0
$$

structured matrix $\Phi$ and a scalar $\gamma>0$ such that for all $\left(h_{d}, \mu_{d}\right) \in \mathcal{V}_{h} \times \mathcal{V}_{\mu}$ the LMIs

$$
\begin{aligned}
\Psi+\Phi+V_{s}\left(h_{d}\right)^{T} \Theta^{T}+\Theta V_{s}\left(h_{d}\right) & <0 \\
P_{0}+h_{d} P_{1} & >0 \\
D_{0}+\sqrt{h_{d}} D_{1} & >0
\end{aligned}
$$

holds where $\beta=\sqrt{h_{\max }}, V_{s}\left(h_{d}\right)=\left[\begin{array}{ccc}\sqrt{h_{d}} I & -I & 0 \\ 0 & -\sqrt{h_{d}} I & -I\end{array}\right]$ and

$$
\begin{aligned}
& \Psi=\left[\begin{array}{ccc}
\Psi_{0} & \Psi_{1 / 2} / 2 & \Psi_{1} / 3 \\
\star & \Psi_{1} / 3 & \Psi_{3 / 2} / 2 \\
\star & \star & 0
\end{array}\right] \\
& \Psi_{0}=\left[\begin{array}{ccccc}
{\left[\bar{A}^{T} P_{0}\right]^{\mathcal{S}}+P_{1} \dot{h}} & 0 & P_{0} E & C^{T} & \bar{A}^{T} D_{0} \\
\star & -D_{0} & 0 & 0 & 0 \\
\star & \star & -\gamma I_{p} & F^{T} & E^{T} D_{0} \\
\star & \star & \star & -\gamma I_{q} & 0 \\
\star & \star & \star & \star & -D_{0}
\end{array}\right]
\end{aligned}
$$

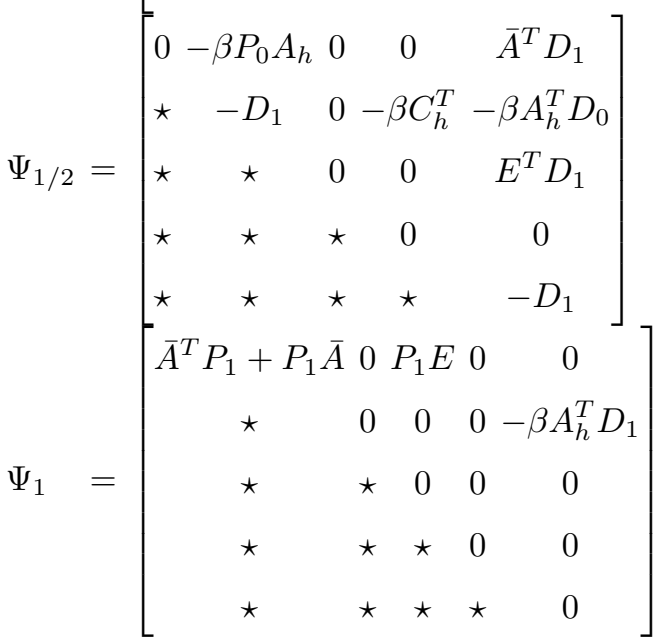

$$
\begin{aligned}
& \Psi_{3 / 2}=\left[\begin{array}{ccccc}
0 & -\beta P_{1} A_{h} & 0 & 0 & 0 \\
\star & 0 & 0 & 0 & 0 \\
\star & \star & 0 & 0 & 0 \\
\star & \star & \star & 0 & 0 \\
\star & \star & \star & \star & 0
\end{array}\right]
\end{aligned}
$$

Proof: Since system (10) is linear in $\sqrt{h(t)}$ then matrices of the form $P(h)=P_{0}+P_{1} h>0$ and $D=D_{0}+D_{1} \sqrt{h}$ are chosen. The reason why $P$ does not involve a term in $\sqrt{h}$ is the non-differentiability of $\sqrt{h}$ for $h=0$. However such a term can be used when the delay is not assumed to take 0 values.

Note that $P(h)>0$ if and only if (12) is satisfied and $D(h)>0$ if and only if (13) using standard convex arguments. Then substitute the explicit expressions of $D(h)$ and $P(h)$ into (8) leads to the expression

$$
\Psi_{0}+\Psi_{1 / 2} \sqrt{h}+\Psi_{1} h+\Psi_{3 / 2} h^{3 / 2}<0
$$

Since the LMI is affine in $\dot{h}$ (only $\Psi_{0}$ depends on $\dot{h}$ ) it is not necessary to include it into the spectral factor $U(\cdot)$. Then computing the spectral factorization of $(17)$ with $U_{s}(h)=$

$$
\begin{aligned}
& {\left[\begin{array}{c}
I \\
\sqrt{h} I \\
h I
\end{array}\right] \text { we get }} \\
& U_{s}^{T}(h)\left[\begin{array}{ccc}
\Psi_{0} & \Psi_{1 / 2} / 2 & \Psi_{1} / 3 \\
\star & \Psi_{1} / 3 & \Psi_{3 / 2} / 2 \\
\star & \star & 0
\end{array}\right] U_{s}(h)<0
\end{aligned}
$$

Applying lemma 2.1 on (18) linearizes the parameter dependence which becomes affine in $\sqrt{h}$. Indeed, we have

$$
V_{s}(h)=\left[\begin{array}{ccc}
\sqrt{h} I & -I & 0 \\
0 & \sqrt{h} I & -I
\end{array}\right]
$$

and $V_{s}(h) U_{s}(h)=0$. Finally, using a convexity argument it suffices to check the negative definiteness of the LMI at the vertices of $H \times U$ (i.e. for every element of $\mathcal{V}_{h} \times \mathcal{V}_{\mu}$ ) to check the feasibility of the LMI over the whole space $H \times U$ and delay and we obtain (11).

\section{Delay-Scheduled state-feedback design}

This section is devoted to the determination of a delayscheduled controller of the form (4) which stabilizes system (1) and ensures $\mathcal{L}_{2}$ performance of the closed-loop system. The closed-loop system obtained from the interconnection of (1) and (4) is governed by the equations 


$$
\left[\begin{array}{ccccc}
-\dot{\hat{h}} \frac{\partial X(\hat{h})}{\partial \hat{h}}+\left[X(\hat{h}) \bar{A}^{T}+Y^{T}(\hat{h}) B_{u}^{T}\right]^{\mathcal{S}} X(\hat{h}) \bar{C}^{T}+Y^{T}(\hat{h}) D_{u}^{T} & -\dot{\hat{h}} \frac{\partial X(\hat{h})}{\partial \hat{h}}+\bar{A} X(\hat{h})+B Y(\hat{h}) & \alpha h A_{h} \tilde{D}(\xi) & E \\
\star & -\gamma I_{q} & \bar{C} X(\hat{h})+D Y(\hat{h}) & h \alpha C_{h} \tilde{D}(\xi) & F \\
\star & \star & -\dot{\hat{h}} \frac{\partial X(\hat{h})}{\partial \hat{h}}-\tilde{D}(\xi) & 0 & 0 \\
\star & \star & \star & -\tilde{D}(\xi) & 0 \\
\star & \star & \star & \star & -\gamma I_{p}
\end{array}\right]<0
$$

$$
\begin{gathered}
\Psi_{0}=\left[\begin{array}{cccccc}
-\left(\mu_{d}+\delta_{\nu}\right) X_{1}+\left[X\left(\delta_{d}\right) \bar{A}^{T}+Y^{T}\left(\delta_{d}\right) B_{u}^{T}\right]^{\mathcal{S}} & X\left(\delta_{d}\right) \bar{C}^{T}+Y^{T}\left(\delta_{d}\right) D_{u}^{T} & -\dot{\delta}_{d} X_{1}+\bar{A} X\left(\delta_{d}\right) & 0 & E \\
\star & -\gamma I_{q} & \bar{C} X\left(\delta_{d}\right) & 0 & F \\
\star & \star & -\left(\mu_{d}+\delta_{\nu}\right) X_{1}-\tilde{D}_{0} & 0 & 0 \\
\star & & \star & \star & -\tilde{D}_{0} & 0 \\
\star & & \star & \star & \star & -\gamma I_{p}
\end{array}\right] \\
\Psi_{1}=\left[\begin{array}{cccccc}
{\left[\bar{A} X_{1}+B_{u} Y_{1}\right]^{\mathcal{S}}} & X_{1} \bar{C}^{T}+Y_{1}^{T} D_{u}^{T} & \bar{A} X_{1} & \beta A_{h} \tilde{D}_{1} & 0 \\
\star & 0 & \bar{C} X_{1} & \beta C_{h} \tilde{D}_{1} & 0 \\
\star & \star & 0 & 0 & 0 \\
\star & \star & \star & 0 & 0 \\
\star & \star & \star & \star & 0
\end{array}\right]
\end{gathered}
$$

$$
\begin{aligned}
\dot{x}(t) & =\bar{A}_{c l}\left(h, \delta_{h}\right) x(t)-\alpha(t) A_{h} w_{0}(t)+E w(t) \\
z(t) & =\bar{C}_{c l}\left(h, \delta_{h}\right) x(t)-\alpha(t) C_{h} w_{0}(t)+F w(t) \\
z_{0}(t) & =\dot{x}(t) \\
w_{0}(t) & =\mathcal{D}_{h}\left(z_{0}(t)\right)
\end{aligned}
$$

with $\hat{h}=h+\delta_{h} \in \hat{H}$. Since the state feedback is of the form $K(\hat{h})$ then the closed-loop system matrices are given by $\bar{A}_{c l}(\hat{h})=A+A_{h}+B_{u} K(\hat{h}), \bar{C}_{c l}(\hat{h})=C+C_{h}+D_{u} K(\hat{h})$.

At this point, several techniques can be employed to compute the controller (4):

(i) either use a change of variable and in this case it is possible to fix a desired form to the controller; or

(ii) elaborate a stabilizability test and deduce a suitable controller either by explicit formulae or implicitly by solving a SDP.

In the present paper we propose a solution based on a change of variable which has several benefits compared to the second one. The main one is the the possibility of choosing the structure of the controller (constant, affine, polynomial or rational). The resulting controller is then implicitly implementable. This is not the case of controllers computed using the second technique (with explicit construction formulae) which may depend on the derivative of the delay, supposed to be unknown. Moreover, the first approach allows for an easy computation of rational controllers through an appropriate choice of the decision matrices.
Theorem 5.1 The system (10) is stabilizable with a delayscheduled state feedback gain $K(\hat{h})=Y(\hat{h}) X^{-1}(\hat{h})$ if there exists a smooth matrix function $X: \hat{H} \rightarrow \mathbb{S}_{++}^{n}$, matrix functions $\tilde{D}: H \times U \times \hat{H} \times \hat{U} \rightarrow \mathbb{S}_{++}^{n}, K: \hat{H} \rightarrow \mathbb{R}^{m \times n}$ and a scalar $\gamma>0$ such that the LMI (14) holds for all $h \in H$, $\dot{h} \in U, \hat{h} \in \hat{H}$ and $\dot{\hat{h}} \in \hat{\Delta}$, where $\xi=\operatorname{col}\left(h, \delta_{h}, \dot{h}, \dot{\delta_{h}}\right)$ and $\alpha=\sqrt{h h_{\max }}$.

Moreover the closed-loop system satisfies $\|z\|_{\mathcal{L}_{2}} /\|w\|_{\mathcal{L}_{2}}<$ $\gamma$.

Proof: The proof is given in Appendix D and is mainly based on the dualization lemma (Scherer and Weiland, 2005).

We develop immediately the following corollary where all the parameter dependent matrices are chosen to be constant:

Corollary 5.1 The system (10) is stabilizable by a constant state feedback $K=Y X^{-1}$ if there exists constant matrices $X, \tilde{D} \in \mathbb{S}_{++}^{n}, Y \in \mathbb{R}^{m \times n}$ and a scalar $\gamma>0$ such that the LMI (20) 


$$
\left[\begin{array}{ccccc}
{\left[X \bar{A}^{T}+Y^{T} B_{u}^{T}\right]^{\mathcal{S}}} & X \bar{C}^{T}+Y^{T} D_{u}^{T} & \bar{A} X & \alpha A_{h} \tilde{D} & E \\
\star & -\gamma I_{q} & \bar{C} X & \alpha C_{h} \tilde{D} & F \\
\star & \star & -\tilde{D} & 0 & 0 \\
\star & \star & \star & -\tilde{D} & 0 \\
\star & \star & \star & \star & -\gamma I_{p}
\end{array}\right]<0
$$

holds for all $h \in \mathcal{V}_{h}$ and $\alpha=\sqrt{h h_{\max }}$.

Moreover the closed-loop system satisfies $\|z\|_{\mathcal{L}_{2}} /\|w\|_{\mathcal{L}_{2}}<$ $\gamma$.

Proof: It is a straightforward application of theorem 5.1 when using constant decision matrices. Then with a convexity argument it is possible to conclude on the feasibility of the LMI over the whole parameter space while checking the feasibility at the vertices of the set $H$ only (i.e. $\mathcal{V}_{h}$ ).

Finally the corollary below stands when the parameter dependent matrices are chosen such that rational controllers of degree 1 in $\hat{h}$ can be designed:

Corollary 5.2 The system (10) is stabilizable with a delayscheduled state feedback $K(\hat{h})=Y(\hat{h}) X(\hat{h})^{-1}$ with $Y(\hat{h})=$ $Y_{0}+Y_{1} \hat{h}$ and $X(\hat{h})=X_{0}+\hat{h} X_{1}$ if there exist matrices $X_{0}, X_{1}, \tilde{D}_{0}, \tilde{D}_{1} \in \mathbb{S}^{n}, Y_{0}, Y_{1} \in \mathbb{R}^{m \times n}$, a full matrix $\Pi$, a $\mathcal{S}^{2}$-structured matrix $\Phi$ and a scalar $\gamma>0$ such that the following $L M I s$

$$
\begin{aligned}
& \Psi+\Phi+\mathcal{K}_{1}^{T}(\xi) \Pi^{T}+\Pi \mathcal{K}_{1}(\xi)<0 \\
& \tilde{D}_{0}+\tilde{D}_{1} \sqrt{h_{d}}>0 \\
& X_{0}+X_{1}\left(h_{d}+\delta_{d}\right)>0
\end{aligned}
$$

hold for all $\xi \in \mathcal{V}_{h} \times \mathcal{V}_{\mu} \times \mathcal{V}_{\delta} \times \mathcal{V}_{\nu}$ with $\mathcal{V}_{\delta}:=\{-\delta, \delta\}, \mathcal{V}_{\nu}:=$ $\left\{\nu_{\min }, \nu_{\max }\right\}, \xi=\left(h_{d}, \mu_{d}, \delta_{d}, \delta_{\nu}\right), \Psi=\left[\begin{array}{cc}\Psi_{0} & \Psi_{1 / 2} / 2 \\ \star & \Psi_{1}\end{array}\right]$ where $\Psi_{0}$ is given by (15), $\Psi_{1}$ by (16) and

$$
\Psi_{1 / 2}=\left[\begin{array}{cccccc}
0 & 0 & 0 & \beta A_{h} \tilde{D}_{0} & 0 \\
\star & 0 & 0 & \beta C_{h} \tilde{D}_{0} & 0 \\
\star \star & -\tilde{D}_{1} & 0 & 0 \\
\star \star & \star & -\tilde{D}_{1} & 0 \\
\star \star & \star & \star & 0
\end{array}\right]
$$

Proof: The proof is similar as for Corollary 4.2. Affine parameter dependent matrices $Y(\hat{h})=Y_{0}+Y_{1} \hat{h}$ and $X(\hat{h})=$ $X_{0}+X_{1} \hat{h}$ are chosen. The matrix $\tilde{D}$ is fixed to be affine in $\sqrt{h(t)}$. Then (14) may be rewritten as

$$
\left[\begin{array}{c}
I \\
\sqrt{h} I
\end{array}\right]^{T} \underbrace{\left[\begin{array}{cc}
\Psi_{0} & \Psi_{1 / 2} / 2 \\
\star & \Psi_{1}
\end{array}\right]}_{\Psi}\left[\begin{array}{c}
I \\
\sqrt{h} I
\end{array}\right]^{T}<0
$$

The linearization procedure is then applied and yields (21). The negative definiteness of $\tilde{D}$ and positive definiteness of $X$ are defined by (22) and (23) respectively.

\section{Example}

We aim to stabilize the following time delay system with time-varying delay

$$
\begin{aligned}
\dot{x}(t)= & {\left[\begin{array}{cc}
-1 & 0 \\
1 & 1
\end{array}\right] x(t)+\left[\begin{array}{ll}
1 & -1 \\
0 & -1
\end{array}\right] x_{h}(t)+\left[\begin{array}{l}
1 \\
0
\end{array}\right] u(t) } \\
& +\left[\begin{array}{l}
1 \\
0
\end{array}\right] w(t) \\
z(t)= & {\left[\begin{array}{ll}
0 & 1 \\
0 & 0
\end{array}\right] x(t)+\left[\begin{array}{l}
0 \\
1
\end{array}\right] u(t) }
\end{aligned}
$$

with $h(t) \in[0.1,0.9],|\dot{h}| \leq 0.2, \dot{\delta}_{h}, \delta_{h} \in[-0.1,0.1]$. Constant scaling (i.e. constant $\tilde{D})$ is chosen and we compute an affine state-feedback of the form $K(\hat{h})=K_{0}+K_{1} \hat{h}$ with

$$
\begin{aligned}
& K_{0}=\left[\begin{array}{ll}
-27.1322 & -17.8448
\end{array}\right] \\
& K_{1}=\left[\begin{array}{ll}
-4.6802 & -3.1368
\end{array}\right]
\end{aligned}
$$

Using this controller, the minimal $\mathcal{L}_{2}$ performance gain is $\gamma=9.89$. It is important to note that, for this example, computing a rational gain does not improve the result and should not be used.

\section{Conclusion}

We have presented in this paper a new model transformation which refines the transformation proposed in (Briat et al., 2007) by allowing the consideration of a larger class of delay values (especially including 0 ). The methodology is similar and is based on the transformation of a timedelay system into an uncertain LPV system where the delay acts as a time-varying parameter. Based on that description, it is possible to propose a new delay-dependent stability lemma based on the full-block $\mathcal{S}$-procedure and derive a constructive approach for the computation of stabilizing state-feedback controllers. Both constant and delayscheduled controllers are considered and uncertainties on the knowledge of the delay are taken into account in the synthesis problem. All the results are given in terms of parameter dependent LMIs depending polynomially on the parameters. These parametrized LMIs are then relaxed into a set of constant LMIs using a linearization result.

\section{Appendix A. Proof of Lemma 2.1}

The proof is a simple application of the Finsler's lemma (Skelton et al., 1997) recalleds hereunder: 
Lemma A.1 Let $M$ by a symmetric matrix and $B$ a matrix of appropriate dimensions, then the following statements are equivalent:

(i) Inequality $\operatorname{Ker}[B]^{T} M \operatorname{Ker}[B]<0$ holds.

(ii) There exists a matrix $N$ of appropriate dimensions such that $M+B^{T} N+N^{T} B<0$

First note that if the quadratic form $U^{T}(\delta) \mathcal{R} U(\delta)$ is added to $U^{T}(\delta) \mathcal{N} U(\delta)$, the expression of the quadratic form remains unchanged (since $\mathcal{R}$ is $\mathcal{S}^{2}$-structured and hence $\left.U^{T}(\delta) \mathcal{R} U(\delta)=0\right)$. However this additional term modifies the eigenvalues the resulting LMI and thus provides extra degrees of freedom. Apply the Finsler's lemma on $U^{T}(\delta) \mathcal{N} U(\delta)<0$ leads to the existence of $\mathcal{Q}(\delta)$ such that

$$
\mathcal{N}+\mathcal{R}+\mathcal{Q}(\delta) V(\delta)+V^{T}(\delta) \mathcal{Q}(\delta)^{T}<0
$$

These parameter dependent LMIs are fully equivalent. Then fixing $\mathcal{Q}$ to be parameter independent (loosing then equivalence) yields the proposed result (7).

\section{Appendix B. Proof of Proposition 3.1}

Let us prove first that for a $\mathcal{L}_{2}$ input signal we get a $\mathcal{L}_{2}$ output signal. Assume that $\eta(t)$ is continuous and denote by $\eta_{p}(t)$ all the signals satisfying $d \eta_{p}(t) / d t=\eta(t)$ then we have

$$
\mathcal{D}_{h}(\eta(t))=\frac{\eta_{p}(t)-\eta_{p}(t-h(t))}{\sqrt{h(t) h_{\max }}}
$$

If $h(t)$ is positive then (B.1) is bounded since $\eta(t)$ is continuous and belongs to $\mathcal{L}_{2}$. Now, let us show that when the delay reaches 0 , the output signal remains bounded. Let us suppose that there exist a (possibly infinite) family of time instants $0 \leq t_{0}<\ldots<t_{i}<t_{i+1}<\ldots$ such that $h\left(t_{i}\right)=0$. Since $\eta_{p}(t)$ is continuously differentiable and hence we have

$$
\lim _{t \rightarrow t_{i}} \frac{\eta_{p}(t)-\eta_{p}(t-h(t))}{\sqrt{h(t) h_{\max }}}=\sqrt{\frac{h\left(t_{i}\right)}{h_{\max }}} \eta\left(t_{i}\right)
$$

Since $\eta(t)$ is continuous and belongs to $\mathcal{L}_{2}$, we can state that $\eta\left(t_{i}\right)$ is always finite and then the output signal satisfies

$$
\lim _{t \rightarrow t_{i}} \frac{\eta_{p}(t)-\eta_{p}(t-h(t))}{\sqrt{h(t) h_{\max }}}=0
$$

This shows that the output signal is bounded if the delay reaches zero. We have shown that the output signal remains bounded for any value of $h(t)$, let us prove now that it has a finite induced $\mathcal{L}_{2}$-norm using a similar method as in $(\mathrm{Gu}$ et al., 2003). We have the following definition

$\left\|\mathcal{D}_{h}(\eta)\right\|_{\mathcal{L}_{2}}^{2}:=\int_{0}^{+\infty} \frac{d t}{h(t) h_{\max }} \int_{t-h(t)}^{t} \eta^{T}(\theta) d \theta \cdot \int_{t-h(t)}^{t} \eta(\theta) d \theta$

Then using the Jensen's inequality (see (Gu et al., 2003)) we obtain

$$
\left\|\mathcal{D}_{h}(\eta)\right\|_{\mathcal{L}_{2}}^{2} \leq \int_{0}^{+\infty} \frac{d t}{h_{\max }} \int_{t-h(t)}^{t} \eta^{T}(\theta) \eta(\theta) d \theta
$$

In order to exhibit the norm of the input signal into the expression, the idea is to exchange the order of integration.

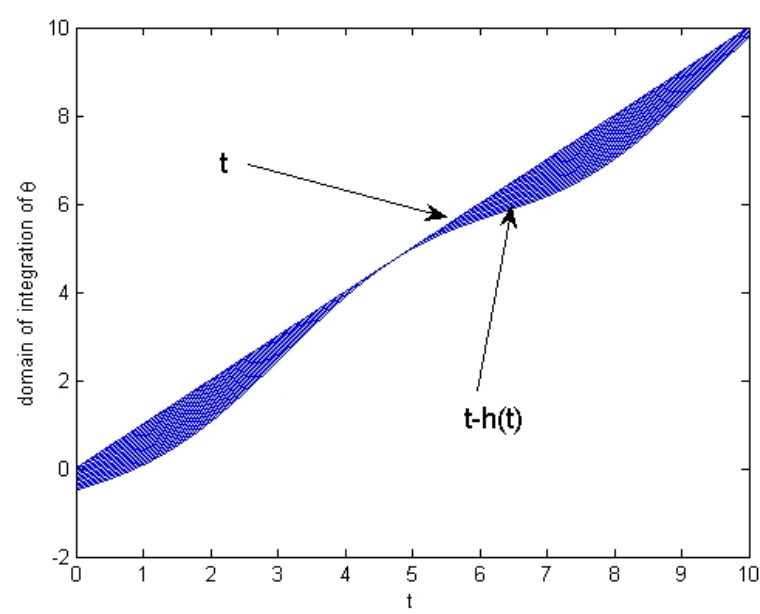

Fig. B.1. Evolution of the domain of integration of (B.2)

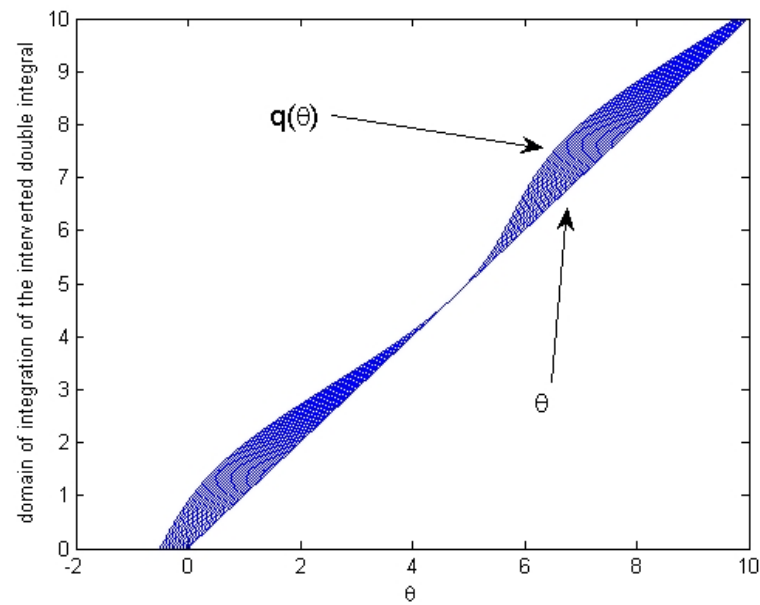

Fig. B.2. Evolution of the domain of integration of (B.3)

The exchange is possible if the dependence between $t$ and $\theta$ is reversed. In the integral equation (B.2), $\theta$ depends on $t$, in the 'exchanged' one $t$ should depend on $\theta$. Note that the integration domain for (B.2) is defined by

$$
\left\{(t, \theta): t \in \mathbb{R}^{+}, \theta \in[t-h(t), t]\right\}
$$

and is represented by the blue/dark surface on Figure B.1.

The exchange is based on the inverse function of $p(t)=$ $t-h(t)$ which is denoted by $q:=p^{-1}$. This inverse function exists since $p(t)$ is strictly increasing according to the assumption $\dot{h}<1$. The inverse function can be seen as the symmetric of $p(t)$ with respect to the axis $\theta=t$ on Figure B.1 and after rotation/flipping of the plane we get the integral domain depicted on Figure B.2 which is formally defined by

$$
\left\{(t, \theta): \theta \in \mathbb{R}^{+}, t \in[\theta, q(\theta)]\right\}
$$

It is easily seen that $t$ depends on $\theta$ and hence the order of integration can be exchanged.

Hence we get 


$$
\begin{aligned}
\left\|\mathcal{D}_{h}(\eta)\right\|_{\mathcal{L}_{2}}^{2} & \leq \frac{1}{h_{\max }} \int_{-h(0)}^{+\infty} \eta^{T}(\theta) \eta(\theta) d \theta \int_{\theta}^{q(\theta)} d t \\
& =\frac{1}{h_{\max }} \int_{-h(0)}^{+\infty} \eta^{T}(\theta) \eta(\theta)(q(\theta)-\theta) d \theta
\end{aligned}
$$

Moreover since the symmetry preserves distances, we have $|q(\theta)-\theta| \leq h_{\max }$ and considering zero initial conditions (i.e. $\eta(s)=0$ for all $s \leq 0$ ) we get

$$
\left\|\mathcal{D}_{h}(\eta)\right\|_{\mathcal{L}_{2}}^{2} \leq\|\eta\|_{\mathcal{L}_{2}}^{2}
$$

stating that $\mathcal{D}_{h}$ defines a $\mathcal{L}_{2}$ input/output stable operator with an $\mathcal{L}_{2}$-induced norm lower than 1 .

\section{Appendix C. Proof of Lemma 4.1}

It is convenient to introduce first a simplified version of the full-block $\mathcal{S}$-procedure (Scherer, 2001):

Lemma C.1 Let us consider system

$$
\begin{aligned}
& \dot{x}(t)=A(t) x(t)+B_{0}(t) w_{0}(t) \\
& z_{0}(t)=C_{0}(t) x(t)+D_{00}(t) w_{0}(t) \\
& w_{0}(t)=\Delta(t) z_{0}(t)
\end{aligned}
$$

and such that the uncertainty $\Delta(t)$ satisfies the integral quadratic constraint (IQC)

$$
\int_{0}^{t} z_{0}(s)^{T}\left[\begin{array}{c}
\Delta(s) \\
I
\end{array}\right]^{T} M_{\Delta}(s)\left[\begin{array}{c}
\Delta(s) \\
I
\end{array}\right] z_{0}(s) d s \geq 0
$$

where $M_{\Delta}(s)$ is a structured symmetric matrix (not necessarily positive semidefinite) and $z_{0} \in \mathcal{L}_{2}$. Then if there exists a matrix $P=P^{T}>0$ and $M_{\Delta}(s)$ such that the following LMI holds (where we omit the dependence on time):

$$
\left[\begin{array}{cc}
\dot{P}+A^{T} P+P A & P B_{0} \\
B_{0}^{T} P & 0
\end{array}\right]+\left[\begin{array}{cc}
0 & C_{0}^{T} \\
I & D_{00}^{T}
\end{array}\right] M_{\Delta}(s)\left[\begin{array}{cc}
0 & I \\
C_{0} & D_{00}
\end{array}\right]<0
$$

then the system (C.1) is asymptotically stable.

The main difficulty is the complete characterization of $\Delta(t)$ in finding a 'good' matrix $M_{\Delta}(s)$ such that (C.2) holds. Indeed, the integral quadratic constraint must be satisfied for every $z_{0}(t) \in \mathcal{L}_{2}$ and all 'trajectories' of the uncertain matrix $\Delta(t)$, meaning that the problem is truly infinite dimensional. Due to this fact, it is not possible (or extremely difficult) to find a matrix $M_{\Delta}(s)$ which totally describes the uncertain operator $\Delta(s)$ especially when $\Delta(s)$ contains dynamic LTV operators. In the remaining of the proof the matrix $M_{\Delta}$ will be chosen as constant.

Let us consider system

$$
\begin{aligned}
& \dot{x}(t)=\bar{A} x(t)-\alpha(t) A_{h} \mathcal{D}_{h}(\dot{x}(t))+E w(t) \\
& z(t)=\bar{C} x(t)-\alpha(t) C_{h} \mathcal{D}_{h}(\dot{x}(t))+F w(t)
\end{aligned}
$$

with $\bar{A}=A+A_{h}, \bar{C}=C+C_{h}$ and $\alpha(t)=\sqrt{h(t) h_{\max }}$. Denoting $w_{0}(t):=\mathcal{D}_{h}(\dot{x}(t)), z_{0}(t)=\dot{x}(t)$ and $\Delta(\cdot):=\mathcal{D}_{h}(\cdot)$. This system exactly falls into the framework of Lemma C.1.
It is possible to extend the approach to deal with robust $\mathcal{H}_{\infty}$ performances by adding the input/output constraint:

$$
\int_{0}^{t}\left[\begin{array}{l}
w(s) \\
z(s)
\end{array}\right]^{T}\left[\begin{array}{cc}
-\gamma I & 0 \\
0 & \gamma^{-1} I
\end{array}\right]\left[\begin{array}{l}
w(s) \\
z(s)
\end{array}\right] d s>0
$$

where $\gamma$ is a positive scalar and we get the LMI

$$
\begin{aligned}
& {\left[\begin{array}{rrc}
\frac{\partial P}{\partial h} \dot{h}+\bar{A}^{T} P(h)+P(h) \bar{A}-\alpha P A_{h} & P E \\
\star & 0 & 0 \\
\star & \star & -\gamma(h, \dot{h}) I
\end{array}\right]} \\
& +\left[\begin{array}{cc}
0 & \bar{A}^{T} \\
I & -\alpha A_{h}^{T} \\
0 & E^{T}
\end{array}\right] \mho(h, \dot{h})\left[\begin{array}{ccc}
0 & I & 0 \\
\bar{A}-\alpha A_{h} & E
\end{array}\right] \\
& +\gamma^{-1}(h, \dot{h})\left[\begin{array}{c}
\bar{C}^{T} \\
-\alpha A_{h}^{T} \\
F^{T}
\end{array}\right]\left[\begin{array}{c}
\bar{C}^{T} \\
-\alpha A_{h}^{T} \\
F^{T}
\end{array}\right]^{T}<0
\end{aligned}
$$

where $\mho(h, \dot{h})$ verifies for all $\eta \in \mathcal{L}_{2}$ the IQC

$$
\int_{0}^{t}\left[\begin{array}{c}
\mathcal{D}_{h}(\eta) \\
I_{n}
\end{array}\right]^{T} \mho(h, \dot{h})\left[\begin{array}{c}
\mathcal{D}_{h}(\eta) \\
I_{n}
\end{array}\right] d s>0
$$

The separator $\mho(h, \dot{h})=\mho^{*}(h, \dot{h})$ is chosen such that it characterizes the $\mathcal{L}_{2}$ induced norm of $\mathcal{D}_{h}$ that is

$$
\int_{0}^{t}\left[\begin{array}{c}
\mathcal{D}_{h}(\eta) \\
I_{n}
\end{array}\right]^{T} \underbrace{\left[\begin{array}{cc}
-1 & 0 \\
0 & 1
\end{array}\right]}_{\mathcal{V}_{1}}\left[\begin{array}{c}
\mathcal{D}_{h}(\eta) \\
I_{n}
\end{array}\right] d s>0
$$

for all $\eta \in \mathcal{L}_{2}$. Hence a set of separators can be parametrized by $\mho=\mho_{1} \otimes D(h, \dot{h})$ where $D(h, \dot{h})=D(h, \dot{h})^{T}>0$ for all $(h, \dot{h}) \in H \times U$. Hence we have

$$
\mho(h, \dot{h}):=\left[\begin{array}{cc}
-D(h, \dot{h}) & 0 \\
\star & D(h, \dot{h})
\end{array}\right]
$$

where $D: H \times U \rightarrow \mathbb{S}_{++}^{n}$. Then expanding (C.6) and performing a Schur complement on quadratic term

$$
-\left[\begin{array}{cc}
\bar{C}^{T} & \bar{A}^{T} D(h, \dot{h}) \\
-\alpha C_{h} & -\alpha A_{h} D(h, \dot{h}) \\
F & E^{T} D(h, \dot{h})
\end{array}\right]\left[\begin{array}{cc}
-\gamma^{-1}(h, \dot{h}) I_{q} & 0 \\
0 & -D^{-1}(h, \dot{h})
\end{array}\right](\star)^{T}
$$

yields inequality (8).

\section{Appendix D. Proof of Theorem 5.1}

First note that the real unknown delay is denoted by $h(t)$ and the estimated one by $\hat{h}(t)=h(t)+\delta_{h}(t)$. Since the controller depends on $X^{-1}$ and $Y$ then both matrices 
should depend on $\hat{h}$ only, which is the only known parameter. On the other hand, since $\tilde{D}$ is not involved in the controller expression, it may depend on any parameter (i.e. $\left.h(t), \delta_{h}(t), \dot{h}(t), \dot{\delta}_{h}(t)\right)$. In what follows, we define the compact notation $\xi=\operatorname{col}\left(h, \delta_{h}, \dot{h}, \dot{\delta_{h}}\right)$ for simplicity.

Note now that LMI (C.6) can be rewritten in the following form

$$
(\star)^{T} M(h, \dot{h}) \underbrace{\left[\begin{array}{ccc}
I & 0 & 0 \\
\bar{A}-\alpha A_{h} & E \\
\hline \begin{array}{c}
\bar{A}^{-} \\
\bar{A}-\alpha A_{h}
\end{array} & E \\
\hline 0 & 0 & I \\
\bar{C} & -\alpha C_{h} & F
\end{array}\right]}_{S^{-}}<0
$$

where $M(h, \dot{h})=\left[\begin{array}{cc}\dot{h} \frac{d P(h)}{d h} & P(h) \\ P(h) & 0\end{array}\right] \oplus \mho(\xi) \oplus\left[-\gamma I_{p}\right] \oplus$ $\left[\gamma^{-1} I_{q}\right]$

In order to provide convex synthesis conditions for the controllers, the multiple products between the closedsystem matrices $\bar{A}_{c l}(\hat{h}), \bar{C}_{c l}(\hat{h})$ and decision matrices $P, D$ must be avoided. It is possible to modify the LMI condition (into an equivalent version) in order to keep one product between system matrices and decision variables only. This is performed by the mean of the dualization lemma (Scherer et al., 1997; Scherer and Weiland, 2004) which is recalled below for completeness:

Lemma D.1 Let $M\left(n_{M}=\operatorname{dim}(M)\right)$ be a nonsingular symmetric matrix with respectively $n^{+}(M)$ and $n^{-}(M)$ positive and negative eigenvalues respectively. Let $\mathcal{S}^{-}=$ $\operatorname{Im}\left(S^{-}\right)$a subspace of dimension $n^{-}(M)$ of $\mathbb{R}^{n_{M}}$ and $\mathcal{S}^{+}=\operatorname{Im}\left(S^{+}\right)$such that $\mathcal{S}^{+}$is the orthogonal complement of $\mathcal{S}^{-}$(i.e. $\left(S^{-}\right)^{T} S^{+}=0$ ).

The following statements are equivalent:

(i) The matrix inequality $\left(S^{-}\right)^{T} M S^{-}<0$ holds.

(ii) The matrix inequality $\left(S^{+}\right)^{T} M^{-1} S^{+}>0$ holds.

First substitute the closed-loop system matrices into (D.1). Note that $\operatorname{dim}(M)=4 n+p+q, n^{-}(M)=2 n+p$ and $\operatorname{rank}\left[S^{-}\right]=2 n+p\left(S^{-}\right.$is defined in (D.1)), where $n^{-}(M)$ is the number of strictly negative eigenvalues of the symmetric matrix $M, n=\operatorname{dim}(x), p=\operatorname{dim}(w)$ and $q=\operatorname{dim}(z)$. Since $n^{-}(M)=\operatorname{rank}\left[S^{-}\right]$then it is possible to apply the dualization lemma and we get

$$
(\star)^{T} M^{-1}(\xi) \underbrace{\left[\begin{array}{ccc}
-\bar{A}_{c l}^{T}(\hat{h}) & -\bar{C}_{c l}^{T}(\hat{h}) & 0 \\
I_{n} & 0 & I_{n} \\
\hline \alpha A_{h}^{T} & \alpha C_{h}^{T} & 0 \\
0 & 0 & -I_{n} \\
\hline-E^{T} & -F^{T} & 0 \\
0 & I_{q} & 0
\end{array}\right]}_{S^{+}}>0
$$

where $M^{-1}(\xi)=\left[\begin{array}{cc}\frac{d P(\hat{h})}{d t} & P(\hat{h}) \\ \star & 0\end{array}\right]^{-1} \oplus \mho^{-1}(\xi) \oplus\left[-\gamma^{-1}(\xi) I_{p}\right] \oplus$

$\left[\gamma(\xi) I_{q}\right]$. Let $X=P^{-1}$, then $\frac{d X(\hat{h})}{d t}=-X \frac{d P(\hat{h})}{d t} X$ and $\left[\begin{array}{cc}\frac{d P(\hat{h})}{d t} & P(\hat{h}) \\ \star & 0\end{array}\right]^{-1}=\left[\begin{array}{cc}0 & X(\hat{h}) \\ \star & \frac{d X(\hat{h})}{d t}\end{array}\right]$. Denote also $\mho^{-1}(\xi)=$
$\left[\begin{array}{cc}-\tilde{D}(\xi) & 0 \\ \star & \tilde{D}(\xi)\end{array}\right]$ with $\tilde{D}(\xi):=D(\xi)^{-1} \in \mathbb{S}_{++}^{n}$. Then expand

(D.2) and noticing that $\tilde{D}(\xi)>0$, the Schur complement can be used on the quadratic term:

$$
-\left[\begin{array}{ccc}
\alpha A_{h} \tilde{D} & E \\
\alpha C_{h} \tilde{D} & F \\
0 & 0
\end{array}\right]\left[\begin{array}{cc}
\tilde{D}(\xi)^{-1} & 0 \\
0 & \gamma^{-1}(\xi) I_{p}
\end{array}\right](\star)^{T}
$$

Finally multiplying the LMI by -1 (to get a negative definite inequality) we obtain inequality (14) in which $Y(\hat{h})=$ $K(\hat{h}) X(\hat{h})$ is a linearizing change of variable. This concludes the proof.

\section{References}

Apkarian, P., Adams, R., 1998. Advanced gain-scheduling techniques for uncertain systems. IEEE Transactions on Automatic Control 6, 21-32.

Ben-Tal, A., Nemirovski, A., 2002. On tractable approximmations of uncertain linear matrix inequalities affected by interval uncertainty. SIAM J. Optim 12(3), 811-833.

Briat, C., Sename, O., Lafay, J.-F., 2007. A LFT $/ \mathcal{H}_{\infty}$ statefeedback design for linear parameter varying time delay systems. In: European Control Conference 2007, Kos, Greece.

Fridman, E., 2006. Stability of systems with uncertain delays: a new 'complete' Lyapunov-Krasovskii Functional. IEEE Transactions on Automatic Control 51, 885-890.

Fridman, E., Shaked, U., 2002. An improved stabilization method for linear time-delay systems. IEEE Transactions on Automatic Control 47(11), 1931-1937. 
Gouaisbaut, F., Peaucelle, D., 2006a. Delay dependent robust stability of time delay-systems. In: $5^{\text {th }}$ IFAC Symposium on Robust Control Design. Toulouse, France.

Gouaisbaut, F., Peaucelle, D., 2006b. Stability of timedelay systems with non-small delay. In: Conference on Decision and Control, San Diego, California.

Gu, K., Kharitonov, V., Chen, J., 2003. Stability of TimeDelay Systems. Birkhäuser.

Jiang, X., Han, Q., 2005. On $\mathcal{H}_{\infty}$ control for linear systems with interval time-varying delay. Automatica 41, 20992106.

Kao, C., Rantzer, A., 2007. Stability analysis of systems with uncertain time-varying delays. Automatica 43, 959970.

Niculescu, S.-I., 2001. Delay effects on stability. A robust control approach. Vol. 269. Springer-Verlag: Heidelbeg.

Roozbehani, M., Knospe, C., 2005. Robust stability and $\mathcal{H}_{\infty}$ performance analysis of interval-dependent timedelay system. In: American Control Conference, Portland, USA.

Sato, M., 2006. Filter design for LPV systems using quadratically parameter-dependent Lyapunov functions. Automatica 42, 2017-2023.

Sato, M., Peaucelle, D., 2007. Robust stability/peformance analaysis for uncertain linear systems via multiple slack variable approach: Polynomial ltipd systems. In: 46rd IEEE Conference on Decision and Control, New Orleans, LA, USA.

Scherer, C. W., 1999. Robust mixed control and LPV control with full-block scalings. Advances in LMI Methods in Control, SIAM.

Scherer, C. W., 2001. LPV control and full-block multipliers. Automatica 37, 361-375.

Scherer, C. W., 2006. LMI relaxations in robust control (to appear). European Journal of Control - Special Issue on 'Linear Matrix Inequalities in Control' (preprint available at http://www.dcsc.tudelft.nl/ cscherer/papers/EJC06.pdf) $12,3-29$.

Scherer, C. W., Gahinet, P., Chilali, M., 1997. Multiobjective output-feedback control via LMI optimization. IEEE Transaction on Automatic Control 42 (7), 896-911.

Scherer, C. W., Weiland, S., 2004. Linear matrix inequalities in control. Tech. rep., Delft Center for Systems and Control (Delft University of Technology) and Department of Electrical Engineering (Eindhoven University of Technology).

Scherer, C. W., Weiland, S., 2005. Linear Matrix Inequalities in Control.

Sename, O., Lafay, J., Rabah, R., 1995. Controllability indices of linear systems with delays. Kybernetika 6, 559580 .

Skelton, R., Iwasaki, T., Grigoriadis, K., 1997. A Unified Algebraic Approach to Linear Control Design. Taylor \& Francis.

Suplin, V., Fridman, E., Shaked, U., 2006. $\mathcal{H}_{\infty}$ control of linear uncertain time-delay systems - a projection ap- proach. IEEE Transactions on Automatic Control 51, 680-685.

Witrant, E., Georges, D., Canudas De Wit, C., Sename, O., 2005. Stabilization of network controlled systems with a predictive approach. In: $1^{\text {st }}$ Workshop on Networked Control System and Fault Tolerant Control, Ajaccio, France.

Xu, S., Lam, J., Zhou, Y., 2006. New results on delaydependent robust $\mathcal{H}_{\infty}$ control for systems with timevarying delays. Automatica 42(2), 343-348.

Zhang, J., Knospe, C., Tsiotras, P., 2001. Stability of time-delay systems: Equivalence between Lyapunov and scaled small-gain conditions. IEEE Transactions on Automatic Control 46, 482-486. 